\title{
Controlling nutritional status is a prognostic factor for patients with lung cancer: a systematic review and meta-analysis
}

\author{
Chi Zhang ${ }^{1 \#}$, Xiao-Kun Li ${ }^{2 \#}$, Zhuang-Zhuang Cong ${ }^{1 \#}$, Chao Zheng ${ }^{2}$, Chao Luo ${ }^{3}$, Kai Xie ${ }^{4}$, Yang Xu ${ }^{4}$, \\ Wen-Feng Gu ${ }^{1}$, Yong Qiang ${ }^{2}$, Yi Shen ${ }^{1,2,3,4}$ \\ ${ }^{1}$ Department of Cardiothoracic Surgery, Jinling Hospital, Medical School of Nanjing University, Nanjing, China; ${ }^{2}$ Department of Cardiothoracic \\ Surgery, Jinling Hospital, School of Medicine, Southeast University, Nanjing, China; ${ }^{3}$ Department of Cardiothoracic Surgery, Jinling Hospital, \\ School of Clinical Medicine, Southern Medical University, Nanjing, China; ${ }^{4}$ Department of Cardiothoracic Surgery, Jinling Hospital, School of \\ Clinical Medicine, Nanjing Medical University, Nanjing, China \\ Contributions: (I) Conception and design: C Zhang; (II) Administrative support: Y Qiang; (III) Provision of study materials or patients: C Zhang, XK \\ Li, ZZ Cong; (IV) Collection and assembly of data: Y Xu; (V) Data analysis and interpretation: Y Xu, WF Gu, C Zhang; (VI) Manuscript writing: All \\ authors; (VII) Final approval of manuscript: All authors. \\ \#These authors contributed equally to this work. \\ Correspondence to: Yi Shen. Department of Cardiothoracic Surgery, Jinling Hospital, Medical School of Nanjing University, Nanjing, China. \\ Email: dryishen@nju.edu.cn; Yong Qiang. Department of Cardiothoracic Surgery, Jinling Hospital, School of Medicine, Southeast University, \\ Nanjing, China. Email: 3947885@qq.com; Xiao-Kun Li. Department of Cardiothoracic Surgery, Jinling Hospital, School of Medicine, Southeast \\ University, Nanjing, China. Email: drlixiaokun@163.com.
}

\begin{abstract}
Background: Nowadays, controlling nutritional status (CONUT) has been used as a prognostic factor in variety of cancers. However, no consensus has been reached on the prognostic value of CONUT in lung cancer. In this study, we aim to investigate the role of CONUT in survival of patients with lung cancer.

Methods: EMBASE, web of science, and Medline were used to search articles in English-language journals. The association between CONUT score and survival of patients with lung cancer was evaluated by using pooled HRs and their 95\% CIs. Chi-square test and I-Square was used to test heterogeneity among studies. Analyses were all performed using Stata 13.0 (Stata Corporation, College Station, TX).

Results: Eight studies with 1,836 patients were eventually included in this meta-analysis. The pooled results showed that high CONUT score had an unfavorable impact on OS (HR =1.63, 95\% CI: 1.30-2.04), DFS (HR $=1.75,95 \%$ CI: 1.35-2.26), CSS (HR =1.45, 95\% CI: 1.01-2.07) and PFS (HR =1.67, 95\% CI: 0.99-2.35), compared with those with low-CONUT.
\end{abstract}

Conclusions: CONUT can be used as a predictor of prognosis in patients with lung cancer. HighCONUT score was significantly associated with poor OS, DFS, CSS and PFS.

Keywords: Controlling nutritional status (CONUT); lung cancer; prognostic factor

Submitted Nov 23, 2020. Accepted for publication Jan 15, 2021.

doi: 10.21037/apm-20-2328

View this article at: http://dx.doi.org/10.21037/apm-20-2328

\section{Introduction}

Lung cancer is a main cause of cancer death worldwide. Despite the development of a variety of treatments, including surgery, radiotherapy and chemotherapy, the prognosis of lung cancer patients is still very poor (1). Even with pathological stage I non-small cell lung cancer,
$10-20 \%$ of patients will relapse and die after undergoing curative surgery (2).

Over the years, people have been studying the prognostic factors of lung cancer. More and more evidence showed that systemic inflammation and malnutrition are related to the poor prognosis of various malignant tumors. So far, many inflammatory and nutritional markers have 
Table 1 The CONUT scoring system

\begin{tabular}{lcccc}
\hline \multirow{2}{*}{ Parameter } & \multicolumn{5}{c}{ Degree } \\
\cline { 2 - 5 } & Normal & Light & Moderate & Severe \\
\hline Serum albumin $(\mathrm{g} / \mathrm{dL})$ & $\geq 3.5$ & $3.0-3.49$ & $2.50-2.99$ & $<2.50$ \\
Score & 0 & 2 & 4 & 6 \\
TC $(\mathrm{mg} / \mathrm{dL})$ & $\geq 180$ & $140-179$ & $100-139$ & $<100$ \\
Score & 0 & 1 & 2 & 3 \\
TLC $\left(/ \mathrm{mm}^{3}\right)$ & $\geq 1,600$ & $1,200-1,599$ & $800-1,199$ & $<800$ \\
Score & 0 & 1 & 2 & 3 \\
\hline
\end{tabular}

CONUT, controlling nutritional status; TC, total serum cholesterol; TLC, total lymphocyte count.

been advanced to predict the prognosis of lung cancer, including neutrophil to lymphocyte ratio (NLR), platelet to lymphocyte ratio (PLR), C-reactive protein to albumin ratio (CAR), lymphocyte to monocyte ratio (LMR) and advanced lung cancer inflammation index (ALI) (3-6).

Controlling nutritional status (CONUT) was first proposed by Ignacio de Ulíbarri et al. based on three parameters, including serum albumin, total lymphocyte count and total cholesterol (7). The scoring system stratifies the CONUT into 4 levels (none/normal: 0; mild/light: 1; moderate: 2; severe: 3) (Table 1). Albumin is considered to be one of the indicators for evaluating human nutritional status, and it can also be used as an acute phase protein to mediate inflammation (8). At present, a large number of studies have confirmed that patients with low albumin level before anti-tumor treatment have poor prognosis. The change of albumin level is closely related to the stage of tumor, and albumin level can reflect the disease progression of patients with malignant carcinoma (9). Lymphocytes play a very important role in tumor immunity. The decrease in the number and the functional defects of lymphocytes indicate a weakened immune system (10). As one of the structural components of cell membrane, cholesterol participates in certain signal pathways being considered necessary for malignant transformation. Many studies have confirmed the relationship between cholesterol and cancer (11). According to recent reports, people with low-density lipoprotein cholesterol levels are more likely to develop tumors (12).

According to the report, CONUT can be used as a prognostic factor to evaluate the prognosis of esophageal cancer, colorectal cancer and other cancers (13-15). However, no consensus has been reached on the prognostic value of CONUT in patients with lung cancer. Thus, we conducted this study to investigate the role of the CONUT score in survival of lung cancer patients. We present the following article in accordance with the PRISMA reporting checklist (available at http://dx.doi.org/10.21037/apm-202328).

\section{Methods}

\section{Search strategy}

EMBASE (OVID, 1990 to October 1, 2020), web of science (1990 to October 1, 2020), and Medline (PubMed, 1990 to October 1,2020) were used. Medical subject heading $(\mathrm{MeSH})$ was used: "Lung Cancer" [Mesh], "controlling nutritional status" [Mesh], "Pulmonary Neoplasms", "Neoplasms, Lung", "Lung Neoplasm", "Neoplasm, Lung", "Neoplasms, Pulmonary", "Neoplasm, Pulmonary", "Pulmonary Neoplasm", "Lung Cancer", "Cancer, Lung", "Cancers, Lung", "Lung Cancers", "Pulmonary Cancer", "Cancer, Pulmonary", "Cancers, Pulmonary", "Pulmonary Cancers", "Cancer of the Lung", "CONUT". Only articles published in English language were included.

\section{Selection criteria}

The eligibility of studies was assessed by two independent reviewers by reviewing titles, abstracts or full text identified by the search. The inclusion criteria were as follows: (I) the patients in the study were diagnosed as lung cancer histologically; (II) serum albumin, total lymphocyte count, and total cholesterol were detected; (III) hazard ratio (HR) and $95 \%$ confidence interval (CI) can be estimated by ample survival data; (IV) Only the newest, largest, or most informative article was included if there were multiple articles based on similar populations. The exclusion criteria were as follows: (I) in animal experiments or vitro studies; (II) review, meeting, comment, editorial, meta-analysis, expert opinion, basic research, and case report; (III) non-English.

\section{Quality assessment and data extraction}

Two reviewers (Chi Zhang and Xiao-Kun Li) assessed the eligible studies, and disagreements were resolved by a third reviewer (Zhuang-Zhuang Cong). Characteristics (first author, publication year, country, number of patients, cut-off values, etc.) were extracted from included studies into results table. The following data were extracted for 
statistical analysis: gender, age, body mass index (BMI), T stage, $\mathrm{N}$ stage, TNM stage, differentiation, smoking, pleural invasion, lymphatic invasion, vascular invasion, surgical procedure, histology, serum carcinoembryonic antigen (CEA), neutrophil-lymphocyte ratio (NLR), performance status and prognostic outcomes (OS, DFS, CSS, PFS). Survival data, including HR, CI, and P value, were extracted from text or tables of the included articles. Only the data of multivariate analysis were extracted when both univariate and multivariate analysis for survival outcome were provided, since the multivariate analysis is more precise compared with univariate analysis. The Newcastle-Ottawa Scale (NOS) was used to assess the quality of the included studies, consisting of three factors: patient selection, comparability of the study groups, and assessment of outcome (16). Studies were assigned using a score of $0-9$, and the high-quality study was defined as a study with quality scores $\geq 6$.

\section{Statistical analysis}

Pooled hazard ratios (HR) and their 95\% confidence intervals $(95 \% \mathrm{CI})$ were used to evaluate the association between CONUT score and survival of lung cancer patients, and pooled odds ratios (OR) and their 95\% CIs were used to evaluate the association between CONUT score and clinicopathological parameters. Statistical parameters were calculated from available numerical data by methods of Parmar et al. (17) when not given directly in a study. Chi-square test and $\mathrm{I}^{2}$ was used to test heterogeneity among studies. When $\mathrm{I}^{2}>50 \%$ or $\mathrm{P}$ value $<0.1$ indicated significant heterogeneity among studies. If heterogeneity was identified among studies, a random effects model was selected to pool the ORs or HRs, otherwise a fixed effects model was performed. By convention, HR $>1$ implied a worse survival outcome in high-CONUT group. Effect of high-CONUT on survival was considered to be statistically significant when $95 \%$ CI for the HR did not overlap 1 . The funnel plot with Egger's weighted regression method and Begg's rank correlation method $(18,19)$ was used to evaluate the publication bias. $\mathrm{P}$ values $<0.05$ were considered as statistical significance. A trim and fill analysis (20) were performed when publication bias was indicated, estimating the number of missing studies (comparisons) in the original dataset and providing a true effect size which has no publication bias. Analyses were all performed using Stata 13.0 (Stata Corporation, College Station, TX).

\section{Results}

\section{Search results}

The search results have been shown in Figure 1. In our study, 318 studies were initially identified from three electronic databases. After screening the title of citations, 173 studies were excluded since they were duplicate studies. After reading the abstracts and full text, 116 of which were excluded for not fulfil the inclusion criteria. After reviewing 29 potentially eligible articles in detail, 8 studies were eventually included in this meta-analysis (21-28).

\section{Study characteristics}

The characteristics of eligible studies are summarized in Table 2. A total of 8 studies published from 2017 to 2020 met the criteria for this meta-analysis. Six studies $(21,23-26,28)$ were conducted in Japan and the remaining two originated from Korea (27) and Turkey (22). All of studies were based on retrospective analysis of the data. The sample sizes of these studies ranged from 32 to 922 patients, with a total of 1,836 patients. Five studies $(23,25-28)$ investigated squamous cell carcinoma and adenocarcinoma, while the remaining three studies investigated only squamous cell carcinoma (24), adenocarcinoma (21) or small cell lung cancer (22). According to cut-off values defined by each study's author for high-CONUT score, 796 patients $(43.4 \%)$ in this meta-analysis had high-CONUT score, ranging from $29.6 \%$ to $67.8 \%$. HRs on overall survival (OS), disease-free survival (DFS), cancer-specific survival (CSS) and progression-free survival (PFS) could be extracted from 7, 4, 2 and 2 of studies, respectively. Since the study by Lee et al. (27) did not provide the original data and only twelve months of Kaplan-Meier survival curves was provided, HR and $95 \%$ CI was not calculated for the accuracy of the conclusion. The Newcastle-Ottawa quality assessment scale (NOS) score for study quality ranged from 6 to 8.

\section{Association between CONUT score and prognosis}

The association between CONUT score and OS was provided in 7 studies (21-26,28), including 914 patients (Figure $2 A)$. Heterogeneity was nonsignificant $\left(\chi^{2}=2.98\right.$, $\left.\mathrm{P}=0.812, \mathrm{I}^{2}<0.1 \%\right)$, thus, a fixed effects model was conducted. The pooled analysis (HR $=1.63,95 \%$ CI: 1.30 2.04; $\mathrm{P}<0.001)$, suggesting that patients with high-CONUT score had shorter OS than those with low-CONUT score. 


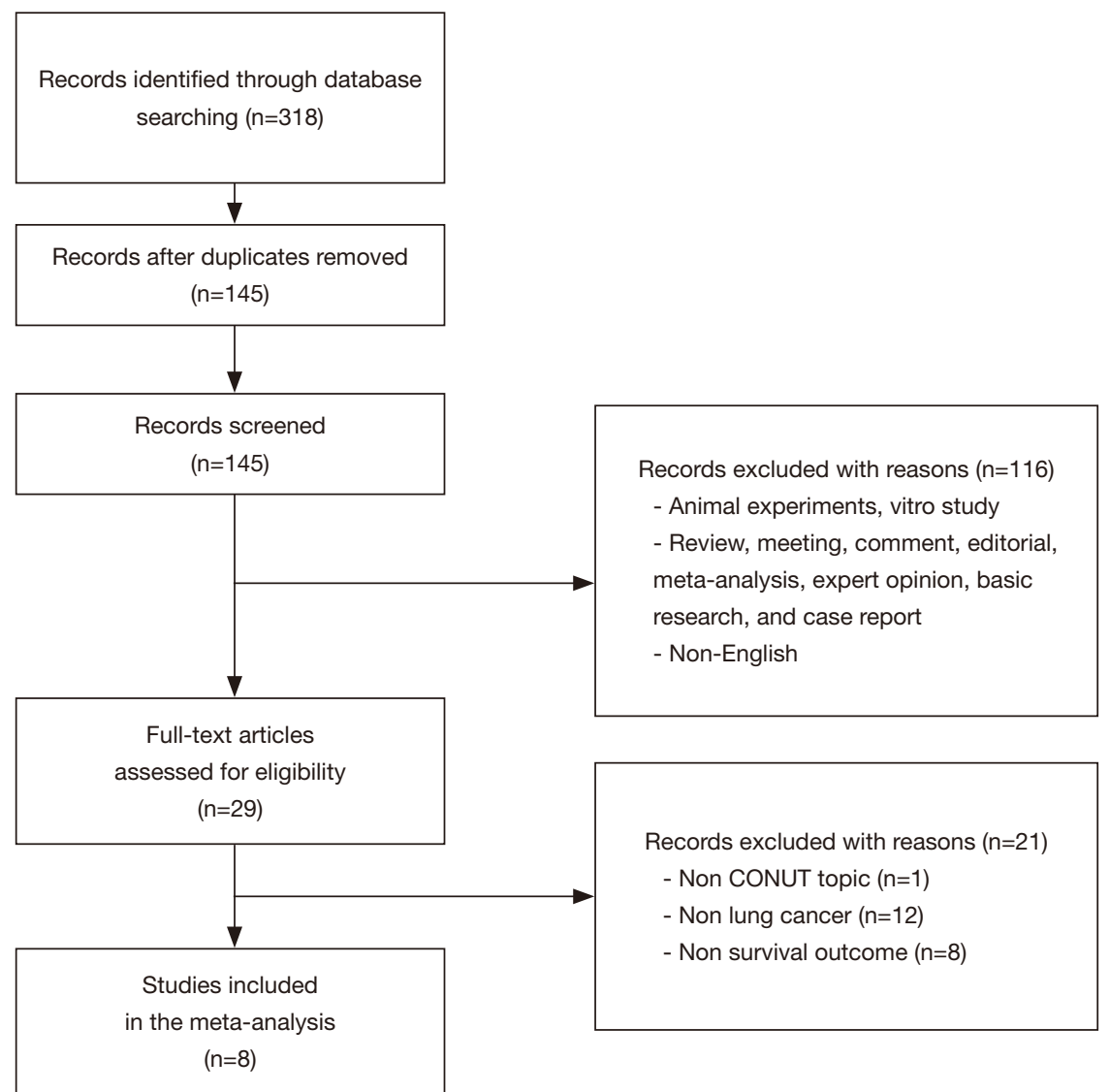

Figure 1 Flow chart.

To investigate the association between CONUT score and DFS, 4 studies $(21,24,25,28)$ with a total of 528 patients were included (Figure $2 B$ ). Heterogeneity was not observed in the analysis $\left(\chi^{2}=2.41, \mathrm{P}=0.544, \mathrm{I}^{2}<0.1 \%\right)$. A fixed effects model was used, and the pooled results showed that patients with low-CONUT score have a better DFS compared with patients with high-CONUT score (HR $=1.75,95 \%$ CI: $1.35-2.26 ; \mathrm{P}<0.001)$.

Two studies $(25,26)$ investigated the association between CONUT score and CSS, including 260 patients (Figure 2C). A fixed effects model was chosen due to heterogeneity was not observed in the analysis $\left(\chi^{2}<0.01, \mathrm{P}=0.961, \mathrm{I}^{2}<0.1 \%\right)$. The pooled HR was 1.45 (95\% CI: 1.01-2.07; $\mathrm{P}=0.045$ ), suggesting that high-CONUT score was significantly associated with worse CSS.

A total of 248 patients in 2 studies $(22,23)$ were evaluated to analyze the correlation between CONUT score and the PFS (Figure 2D). Pooled results indicated an association between a high CONUT score and poor PFS $(\mathrm{HR}=1.67$, 95\% CI: $0.99-2.35 ; \mathrm{P}<0.001)$. For no heterogeneity was observed $\left(\chi^{2}=5.15, \mathrm{P}=0.254, \mathrm{I}^{2}=23 \%\right)$, a fixed effects model was used for the analysis.

\section{Association between CONUT score and clinicopathological characteristics}

We investigated the association between CONUT score and the clinicopathological characteristics of patients with lung cancer. Pooled results showed that CONUT score was high in elderly patients [odds ratio $(\mathrm{OR})=1.47,95 \%$ CI: $1.18-1.82 ; \mathrm{P}<0.001]$, male patients $(\mathrm{OR}=1.47,95 \%$ CI: $1.18-1.82 ; \mathrm{P}=0.001)$, high-stage $(\mathrm{OR}=0.51,95 \%$ CI: $0.40-0.65 ; \mathrm{P}=0.001)$, smoking $(\mathrm{OR}=1.61,95 \% \mathrm{CI}$ : 1.31-1.97; $\mathrm{P}<0.001$ ), abnormal level of preoperative CEA $(\mathrm{OR}=0.43,95 \%$ CI: $0.25-0.73 ; \mathrm{P}=0.002)$ and NLR (OR $=3.91,95 \%$ CI: $2.28-6.72 ; \mathrm{P}<0.001)$. However, we detected no significant associations between CONUT score and BMI (OR =0.32, 95\% CI: 0.09-1.16; $\mathrm{P}=0.084)$, T stage $(\mathrm{OR}=0.63,95 \% \mathrm{CI}: 0.40-1.01 ; \mathrm{P}=0.055), \mathrm{N}$ stage $(\mathrm{OR}$ $=0.65,95 \%$ CI: $0.33-1.30 ; \mathrm{P}=0.222)$, differentiation $(\mathrm{OR}$ 
Table 2 Characteristics of the selected studies included in the meta-analysis

\begin{tabular}{|c|c|c|c|c|c|c|c|c|c|c|}
\hline Author & Year & Country & Study type & $\begin{array}{c}\text { Number } \\
\text { (male/female) }\end{array}$ & $\begin{array}{l}\text { Mean age } \\
\text { (range) }\end{array}$ & $\begin{array}{l}\text { Tumor } \\
\text { stage }\end{array}$ & Cut-off & CONUT score & \multicolumn{2}{|c|}{ Outcome Quality } \\
\hline $\begin{array}{l}\text { Akamine et al. } \\
\text { (21) }\end{array}$ & 2017 & Japan & $\begin{array}{l}\text { Retrospective, } \\
\text { single center }\end{array}$ & 109 [76/33] & 72 [45-85] & $\begin{array}{l}\text { I: } 74 ; \text { II: 24; } \\
\text { III: 9; IV: } 2\end{array}$ & $\geq 1$ & $\begin{array}{c}0: 35(32.1 \%) ; \geq 1: 74 \\
(67.8 \%)\end{array}$ & OS; DFS & 8 \\
\hline Shoji et al. (26) & 2017 & Japan & $\begin{array}{l}\text { Retrospective, } \\
\text { single center }\end{array}$ & $138[79 / 59]$ & 68 [37-86] & I: 138 & $\geq 1$ & $\begin{array}{c}0: 59(42.8 \%) ; \geq 1: 79 \\
(57.2 \%)\end{array}$ & $\begin{array}{l}\text { CSS; OS; } \\
\text { RFS }\end{array}$ & 7 \\
\hline Ohba et al. (23) & 2019 & Japan & $\begin{array}{l}\text { Retrospective, } \\
\text { single center }\end{array}$ & 32 [29/3] & 65 [44-85] & III; IV & $\geq 3$ & $\begin{array}{l}\leq 2: 22(68.8 \%) \\
\geq 3: 10(31.2 \%)\end{array}$ & OS; PFS & 7 \\
\hline $\begin{array}{l}\text { Takamori et al. } \\
\text { (28) }\end{array}$ & 2019 & Japan & $\begin{array}{l}\text { Retrospective, } \\
\text { single center }\end{array}$ & $189[113 / 76]$ & 68 [29-93] & $\begin{array}{c}\text { I [145]; } \geq \text { II } \\
{[44]}\end{array}$ & $\geq 2$ & $\begin{array}{l}\leq 1: 127(67.2 \%) \\
\geq 2: 62(32.8 \%)\end{array}$ & OS; DFS & 8 \\
\hline Yılmaz et al. (22) & 2020 & Turkey & $\begin{array}{l}\text { Retrospective, } \\
\text { single center }\end{array}$ & 216 [184/32] & 61 [36-83] & $\begin{array}{c}\text { I-III [59]; IV } \\
\text { [157] }\end{array}$ & $\geq 2$ & $\begin{array}{l}\leq 1: 89(41.3 \%) \\
\geq 2: 127(58.7 \%)\end{array}$ & OS; PFS & 7 \\
\hline
\end{tabular}

a, score from a maximum of 9 evaluated by the Newcastle-Ottawa quality assessment scale for cohort studies. CONUT, controlling nutritional status; OS, overall survival; DFS, disease-free survival; CSS, cancer-specific survival; PFS, progression-free survival; RFS, recurrence-free survival.

$=0.92,95 \%$ CI: $0.47-1.79 ; \mathrm{P}=0.81)$, pleural invasion $(\mathrm{OR}$ $=0.92,95 \%$ CI: $0.47-1.79 ; \mathrm{P}=0.81)$, lymphatic invasion $(\mathrm{OR}$ $=1.25,95 \%$ CI: $0.74-2.11 ; \mathrm{P}=0.398)$, lymphatic invasion ( $\mathrm{OR}=1.11,95 \%$ CI: $0.54-2.29 ; \mathrm{P}=0.783)$, vascular invasion $(\mathrm{OR}=1.45,95 \% \mathrm{CI}: 0.90-2.36 ; \mathrm{P}=0.13)$, surgical procedure $(\mathrm{OR}=1.02,95 \%$ CI: 0.74-1.39; $\mathrm{P}=0.926)$, Histology (OR $=0.78,95 \%$ CI: $0.37-1.66 ; \mathrm{P}=0.52$ ), and performance status $(\mathrm{OR}=0.90,95 \%$ CI: $0.36-2.27 ; \mathrm{P}=0.826)$. Heterogeneity was observed in the analysis of the relationships between CONUT score and $\mathrm{BMI}\left(\mathrm{P}<0.001, \mathrm{I}^{2}=87.6 \%\right)$ and histology $\left(\mathrm{P}=0.007, \mathrm{I}^{2}=75.5 \%\right)$, therefore, a random effects model was used. The other assessments were performed using a fixed effects model (Table 3).

\section{Cut-off values of CONUT score}

Three studies $(21,25,26)$ used 1 as cut-off, including 369 patients. The pooled HR was 1.55 (95\% CI: $1.10-2.18$, $\mathrm{P}=0.012)$. Three studies $(22,24,28)$ used 2 as cut-off including 513 patients (HR $=1.63,95 \%$ CI: 1.21-2.21, $\mathrm{P}=0.002$ ). These two subgroup analyses both observed that high-CONUT score was associated with unfavorable prognostic outcomes regardless of cut-off value.

\section{Publication bias}

There was publication bias for OS (Egger test, $\mathrm{P}=0.023$ ) and DFS (Egger test, $\mathrm{P}=0.013$ ). The trim and fill analysis suggested that 2 studies (comparisons) were missing from our datasets respectively (marked with a square border in Fig.3a and Fig.3b). Nevertheless, reported significant effects of CONUT on the survival outcomes were complete (OS: $\mathrm{HR}=1.553,95 \%$ CI: $1.251-1.929$; DFS: HR $=1.581,95 \%$ CI: 1.265-1.976) after adding those missing data to the original datasets, suggesting that the impact of publication bias on the overall results was negligible.

\section{Discussion}

In recent years, many researchers have focused on inflammation and nutritional status in various solid tumor for growing evidence has proven it a key role in the carcinogenesis, progression, and metastasis of cancer (29-32). It has been reported that CONUT can be used as an index to evaluate the prognosis of many solid cancers. Takagi et al. (33) conducted a meta-analysis investigating the prognostic value of CONUT in hepatocellular 

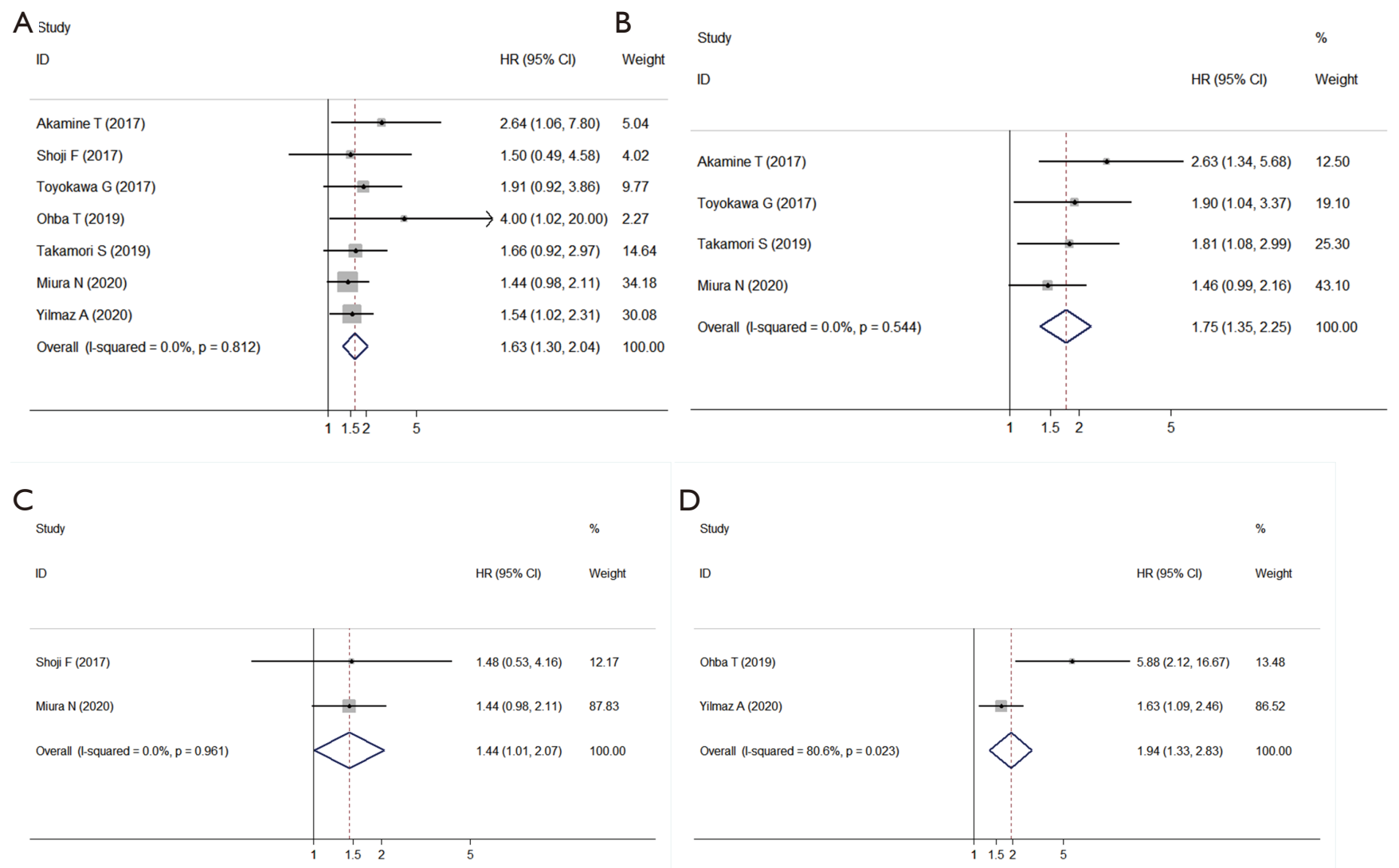

Figure 2 Forest plots demonstrating primary endpoint in terms of high controlling nutritional status (CONUT) group versus low CONUT group. (A) Overall survival; (B) disease-free survival; (C) cancer-specific survival; (D) progression-free survival.

carcinoma (HCC) patients undergoing hepatectomy. The results demonstrated that high-CONUT has a poor impact on OS and RFS in HCC patients. Takagi et al. $(34,35)$ also conducted another two meta-analyses exploring the prognostic value of CONUT in gastric and colorectal cancer. The results revealed that both gastric and colorectal cancer patients with a high-COUNT score had reduced OS, CSS and RFS. Toyokawa et al. (36) introduced the CONUT as a prognostic factor in patients undergoing curative thoracoscopic esophagectomy. They found that the patients with low-CONUT yielded significantly longer OS and RFS compared with high-COUNT group. Huang et al. (37) confirmed that CONUT score was a prognostic factor for breast cancer patients. The results showed that low-CONUT yielded significantly longer OS and RFS compared with high-COUNT group. However, the relationship between CONUT score and the prognosis of patients with lung cancer remains controversial. As we know, this systematic review and meta-analysis is the first study of the prognostic significance of the CONUT score in lung cancer patients. And the results showed that high-CONUT score has unfavorable prognostic outcomes in patients with lung cancer.

Our meta-analysis included 8 studies with 1,836 patients and illustrated that high CONUT score has an unfavorable impact on OS (HR =1.63, 95\% CI: 1.30-2.04), DFS (HR $=1.75,95 \%$ CI: $1.35-2.26)$, CSS $(\mathrm{HR}=1.45,95 \%$ CI: 1.01-2.07) and PFS (HR $=1.67,95 \%$ CI: 0.99-2.35). However, Miura et al. (25) proved no statistical differences both in DFS $(\mathrm{P}=0.9238)$ and CSS $(\mathrm{P}=0.8661)$ in KaplanMeier analysis. Takamori et al. (28) found that CONUT score was not an independent prognostic factor for DFS (HR =1.66, 95\% CI: 0.92-2.97; $\mathrm{P}=0.088$ ). Several probable reasons for these inconsistent results have been speculated. One reason may be that the cut-off values adopted were different though all the investigators used Receiver operating characteristics curve (ROC) to determine the optimal cut-off value of CONUT. A study by Akamine 
Table 3 Relationships between CONUT score and clinicopathological features

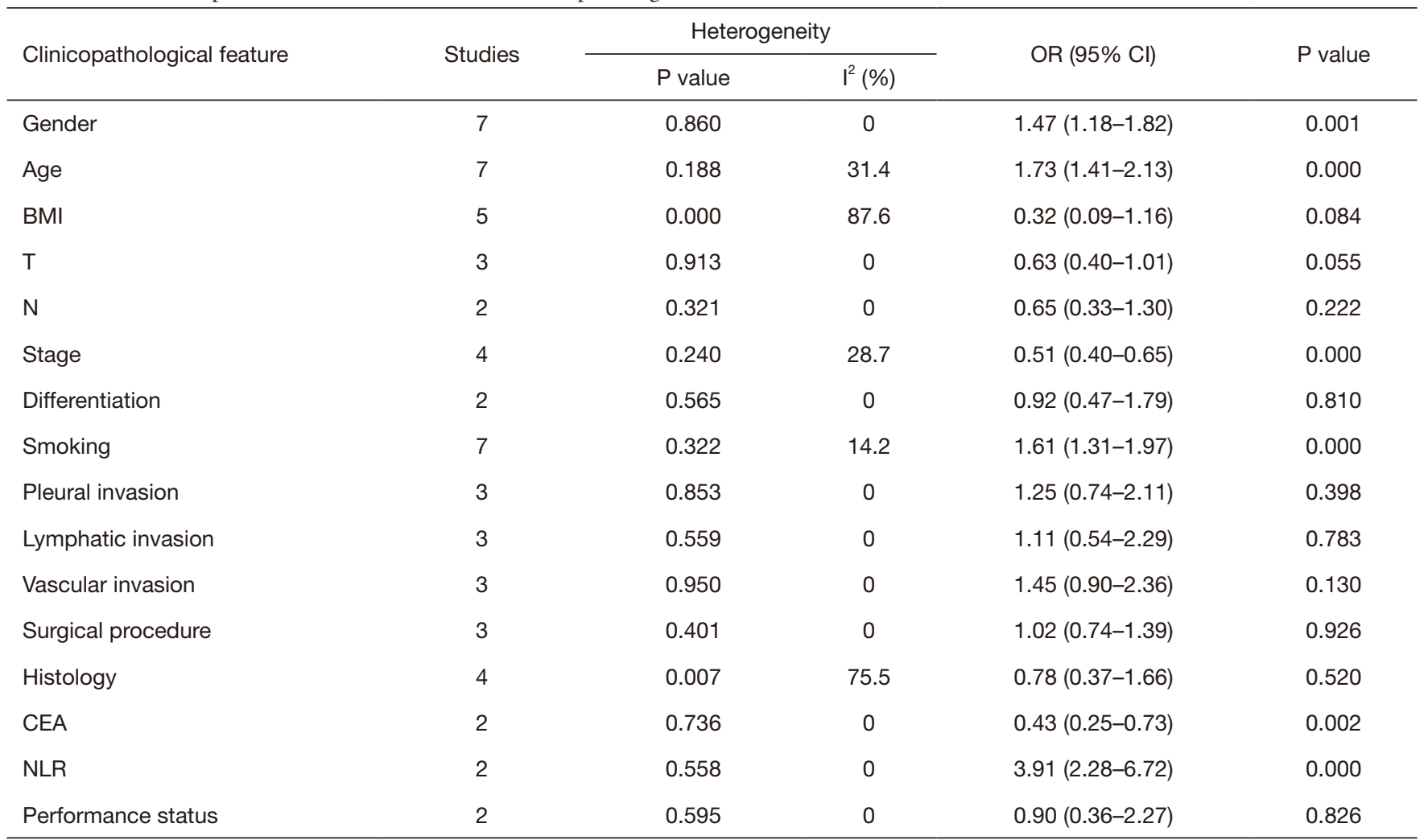

CONUT, controlling nutritional status; OR, odds ratio; BMI, body mass index; T, pathological T status; N, pathological N status; CEA, carcinoembryonic antigen; NLR, neutrophil-to-lymphocyte ratio.
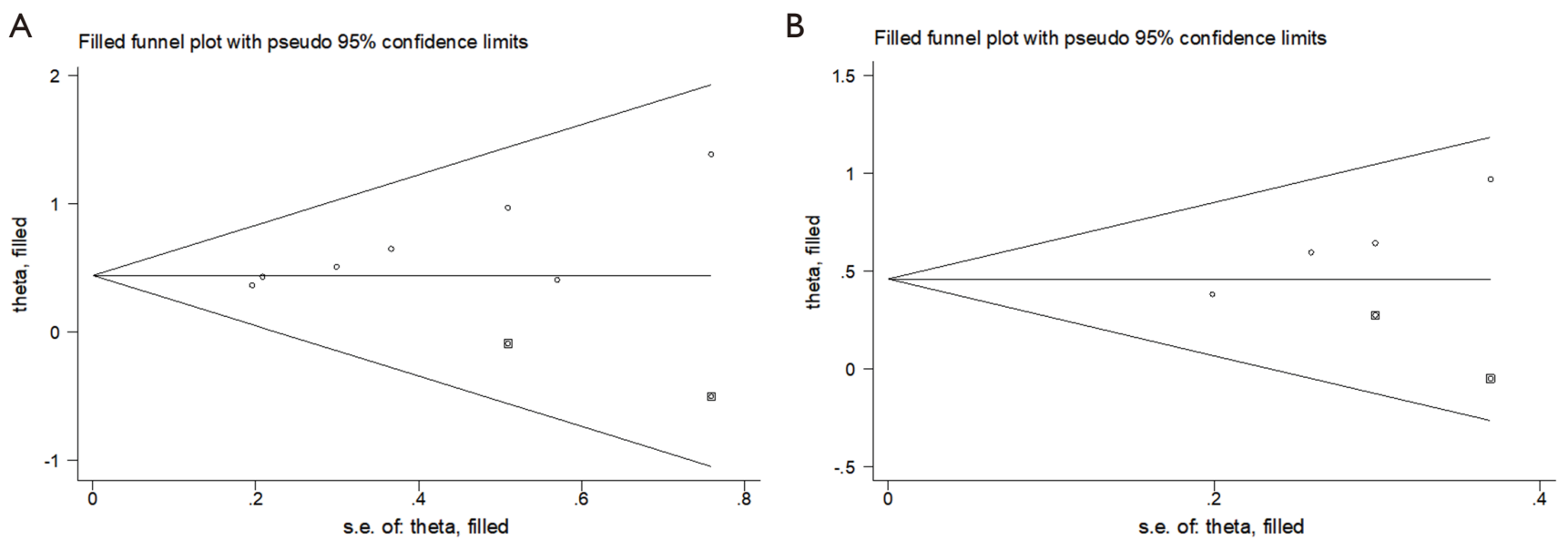

Figure 3 A funnel plot to assess potential publication bias. The circles and squares represent observed data and data added by the trim-andfill analysis (see the main text). (A) Funnel plot of overall survival; (B) funnel plot of disease-free survival. 
et al. (21) demonstrated no significant association between the CONUT and the DFS and OS when the cut-off score was set at 2. Contrary to Akamine's conclusion, other studies $(22,24,28)$ using 2 as the cut-off score confirmed the consistent prognostic value of CONUT. Moreover, the clinicopathological characteristics of each study were also different. Akamine's study only assessed patients with lung adenocarcinoma with obstructive lung disease, Shoji's study (26) only analyzed clinicopathological features of pathological stage I non-small cell lung cancer (NSCLC) patients, Ohba's (23) study only included patients with advanced NSCLC who received pembrolizumab monotherapy, Miura's (25) study only contained Elderly NSCLC patients.

Analysis in subgroup of different cut-off values was also performed. Both of the subgroup analysis showed significant differences [1 (HR $=1.55,95 \%$ CI: 1.10-2.18; $\mathrm{P}=0.012$ ); 2 (HR: 1.63, 95\% CI: 1.21-2.21; $\mathrm{P}=0.002$ )]. Besides, the study by Ohba et al. (23) used 3 as cut off value (HR $=4.00,95 \%$ CI: $1.02-20 ; \mathrm{P}=0.048)$ also indicated significant differences. There is not much variation between the results produced using different cut-off values, and more research is needed to get uniform standards.

The biological mechanism explaining the correlation between the CONUT score and outcomes has not been adequately researched. Each component of the CONUT score is related to the prognosis of patients with lung cancer. The level of total lymphocyte count reflects the body's immune level and the development of tumorigenesis (38). Serum albumin itself is the main indicator of nutritional status, and is also the acute phase protein mediating inflammatory response (8). The production of albumin can also be regulated by inflammatory factors such as IL-6 or hormones (39). The change of albumin level is closely related to tumor stage, that is, albumin levels can reflect the progression of disease in lung cancer patients (40). Cholesterol is essential for maintaining the integrity, fluidity, and function of cell membranes and safeguarding signal transduction. Serum cholesterol levels have been reported to correlate with lung cancer progression and survival (41). In our analysis, we also noted that high CONUT score was significantly associated with elderly patients, male patients, advanced TNM stages, smoking and abnormal preoperative serum CEA, NLR level. These clinicopathological features are recognized as significant factors in the poor prognosis of cancer patients, providing evidence for the scientific validity of CONUT on the other hand.

\section{Limitation}

All the studies included were from Asia. Moreover, the cut-off value used to evaluate CONUT score may lack sensitivity and yield false-negative results, and there is no unified standard up to now. Although we used the trim and fill analysis when there is publication bias, the method failed to consider the other causes of funnel plot asymmetry except publication bias. The reason may be related to the number of included articles, the source of the studies, the clinical features of the patients included, types of research and so on. The existence of these defects indicates the necessity for more high-quality studies exploring the correlation of CONUT with survival outcomes in patients with lung cancer.

\section{Conclusions}

CONUT can be used as a predictor of prognosis in patients with lung cancer. High-CONUT score was significantly associated with poor OS, DFS, CSS and PFS.

\section{Acknowledgments}

The authors would like to acknowledge the support of all colleagues in Department of Cardiothoracic Surgery in Jinling Hospital.

Funding: This work was supported by the National Natural Science Foundation of China (81172032) and the Natural Science Foundation of Jiangsu Province (BK20181239).

\section{Footnote}

Reporting Checklist: The authors have completed the PRISMA reporting checklist. Available at http://dx.doi.org/10.21037/ apm-20-2328

Peer Review File: Available at http://dx.doi.org/10.21037/ apm-20-2328

Conflicts of Interest: All authors have completed the ICMJE uniform disclosure form (available at http://dx.doi. org/10.21037/apm-20-2328). The authors have no conflicts of interest to declare.

Ethical Statement: The authors are accountable for all aspects of the work in ensuring that questions related 
to the accuracy or integrity of any part of the work are appropriately investigated and resolved.

Open Access Statement: This is an Open Access article distributed in accordance with the Creative Commons Attribution-NonCommercial-NoDerivs 4.0 International License (CC BY-NC-ND 4.0), which permits the noncommercial replication and distribution of the article with the strict proviso that no changes or edits are made and the original work is properly cited (including links to both the formal publication through the relevant DOI and the license). See: https://creativecommons.org/licenses/by-nc-nd/4.0/.

\section{References}

1. Torre LA, Bray F, Siegel RL, et al. Global cancer statistics, 2012. CA Cancer J Clin 2015;65:87-108.

2. Sawabata N, Miyaoka E, Asamura H, et al. Japanese lung cancer registry study of 11,663 surgical cases in 2004: demographic and prognosis changes over decade. J Thorac Oncol 2011;6:1229-35.

3. Şahin F, Aslan AF. Relationship between Inflammatory and Biological Markers and Lung Cancer. J Clin Med 2018;7:160.

4. Mandaliya H, Jones M, Oldmeadow C, et al. Prognostic biomarkers in stage IV non-small cell lung cancer (NSCLC): neutrophil to lymphocyte ratio (NLR), lymphocyte to monocyte ratio (LMR), platelet to lymphocyte ratio (PLR) and advanced lung cancer inflammation index (ALI). Transl Lung Cancer Res 2019;8:886-94.

5. Diem S, Schmid S, Krapf M, et al. Neutrophil-toLymphocyte ratio (NLR) and Platelet-to-Lymphocyte ratio (PLR) as prognostic markers in patients with nonsmall cell lung cancer (NSCLC) treated with nivolumab. Lung Cancer 2017;111:176-81.

6. Karki R, Man SM, Kanneganti TD. Inflammasomes and Cancer. Cancer Immunol Res 2017;5:94-9.

7. Ignacio de Ulíbarri J, Gonzalez-Madrono A, de Villar NG, et al. CONUT: a tool for controlling nutritional status. First validation in a hospital population. Nutr Hosp 2005;20:38-45.

8. $\mathrm{Wu} \mathrm{N}$, Chen $\mathrm{G}, \mathrm{Hu} \mathrm{H}$, et al. Low pretherapeutic serum albumin as a risk factor for poor outcome in esophageal squamous cell carcinomas. Nutr Cancer 2015;67:481-5.

9. Li J, Cheng Y, Liu G, et al. The association of pretreatment serum albumin with outcomes in bladder cancer: a meta-analysis. Onco Targets Ther 2018;11:3449-59.
10. Junttila MR, de Sauvage FJ. Influence of tumour microenvironment heterogeneity on therapeutic response. Nature 2013;501:346-54.

11. Tomita M, Ayabe T, Shimizu T, et al. Preoperative Total Serum Cholesterol and Patients' Survival in Resected Nonsmall Cell Lung Cancer. Lung Cancer Int 2012;2012:463520.

12. Kuzu OF, Noory MA, Robertson GP. The Role of Cholesterol in Cancer. Cancer Res 2016;76:2063-70.

13. Yoshida N, Harada K, Baba Y, et al. Preoperative controlling nutritional status (CONUT) is useful to estimate the prognosis after esophagectomy for esophageal cancer. Langenbecks Arch Surg 2017;402:333-41.

14. Kuroda D, Sawayama H, Kurashige J, et al. Controlling Nutritional Status (CONUT) score is a prognostic marker for gastric cancer patients after curative resection. Gastric Cancer 2018;21:204-12.

15. Ahiko Y, Shida D, Horie T, et al. Controlling nutritional status (CONUT) score as a preoperative risk assessment index for older patients with colorectal cancer. BMC Cancer 2019;19:946.

16. Stang A. Critical evaluation of the Newcastle-Ottawa scale for the assessment of the quality of nonrandomized studies in meta-analyses. Eur J Epidemiol 2010;25:603-5.

17. Parmar MK, Torri V, Stewart L. Extracting summary statistics to perform meta-analyses of the published literature for survival endpoints. Stat Med 1998;17:2815-34.

18. Begg CB, Mazumdar M. Operating characteristics of a rank correlation test for publication bias. Biometrics 1994;50:1088-101.

19. Egger M, Davey Smith G, Schneider M, et al. Bias in meta-analysis detected by a simple, graphical test. BMJ 1997;315:629-34.

20. Duval S, Tweedie R. Trim and fill: A simple funnel-plotbased method of testing and adjusting for publication bias in meta-analysis. Biometrics 2000;56:455-63.

21. Akamine T, Toyokawa G, Matsubara T, et al. Significance of the Preoperative CONUT Score in Predicting Postoperative Disease-free and Overall Survival in Patients with Lung Adenocarcinoma with Obstructive Lung Disease. Anticancer Res 2017;37:2735-42.

22. Yilmaz A, Tekin SB, Bilici M, et al. The Significance of Controlling Nutritional Status (CONUT) Score as a Novel Prognostic Parameter in Small Cell Lung Cancer. Lung 2020;198:695-704.

23. Ohba T, Takamori S, Toyozawa R, et al. Prognostic impact of the Controlling Nutritional Status score in patients with 
non-small cell lung cancer treated with pembrolizumab. J Thorac Dis 2019;11:3757-68.

24. Toyokawa G, Kozuma Y, Matsubara T, et al. Prognostic impact of controlling nutritional status score in resected lung squamous cell carcinoma. J Thorac Dis 2017;9:2942-51.

25. Miura N, Shoji F, Kozuma Y, et al. Preoperative Immune-Nutritional Abnormality Predicts Poor Outcome in Elderly Non-Small-Cell Lung Cancer Patients with Comorbidities. Ann Thorac Cardiovasc Surg 2020;26:240-7.

26. Shoji F, Haratake N, Akamine T, et al. The Preoperative Controlling Nutritional Status Score Predicts Survival After Curative Surgery in Patients with Pathological Stage I Non-small Cell Lung Cancer. Anticancer Res 2017;37:741-7.

27. Lee SC, Lee JG, Lee SH, et al. Prediction of postoperative pulmonary complications using preoperative controlling nutritional status (CONUT) score in patients with resectable non-small cell lung cancer. Sci Rep 2020;10:12385.

28. Takamori S, Toyokawa G, Shimokawa M, et al. A novel prognostic marker in patients with non-small cell lung cancer: musculo-immuno-nutritional score calculated by controlling nutritional status and creatine kinase. J Thorac Dis 2019;11:927-35.

29. Souza Cunha M, Wiegert EVM, Calixto-Lima L, et al. Relationship of nutritional status and inflammation with survival in patients with advanced cancer in palliative care. Nutrition 2018;51-52:98-103.

30. Ni XC, Xu J, Yi Y, et al. Inflammation-nutrition score predicts prognosis of patients with resectable hepatocellular carcinoma. Int J Clin Oncol 2019;24:825-35.

31. Zhu Y, Li JH, Yang J, et al. Inflammation-nutrition scope predicts prognosis of early-stage hepatocellular carcinoma after curative resection. Medicine (Baltimore) 2017;96:e8056.

32. Galizia G, Auricchio A, de Vita F, et al. Inflammatory and nutritional status is a predictor of long-term outcome in patients undergoing surgery for gastric cancer. Validation of the Naples prognostic score. Ann Ital Chir 2019;90:404-16.

33. Takagi K, Domagala P, Polak WG, et al. Prognostic significance of the controlling nutritional status (CONUT) score in patients undergoing hepatectomy for hepatocellular carcinoma: a systematic review and meta- analysis. BMC Gastroenterol 2019;19:211.

34. Takagi K, Domagala P, Polak WG, et al. Prognostic significance of the controlling nutritional status (CONUT) score in patients undergoing gastrectomy for gastric cancer: a systematic review and meta-analysis. BMC Surg 2019;19:129.

35. Takagi K, Buettner S, Ijzermans JNM. Prognostic significance of the controlling nutritional status (CONUT) score in patients with colorectal cancer: A systematic review and meta-analysis. Int J Surg 2020;78:91-6.

36. Toyokawa T, Kubo N, Tamura T, et al. The pretreatment Controlling Nutritional Status (CONUT) score is an independent prognostic factor in patients with resectable thoracic esophageal squamous cell carcinoma: results from a retrospective study. BMC Cancer 2016;16:722.

37. Huang ZZ, Song CG, Huang JJ, et al. Prognostic significance of the Controlling Nutritional Status (CONUT) score in surgically treated breast cancer patients. Gland Surg 2020;9:1370-9.

38. Suzuki R, Wei X, Allen PK, et al. Prognostic Significance of Total Lymphocyte Count, Neutrophilto-lymphocyte Ratio, and Platelet-to-lymphocyte Ratio in Limited-stage Small-cell Lung Cancer. Clin Lung Cancer 2019;20:117-23.

39. Castell JV, Gomez-Lechon MJ, David M, et al. Acutephase response of human hepatocytes: regulation of acutephase protein synthesis by interleukin-6. Hepatology 1990;12:1179-86.

40. Miura K, Hamanaka K, Koizumi T, et al. Clinical significance of preoperative serum albumin level for prognosis in surgically resected patients with nonsmall cell lung cancer: Comparative study of normal lung, emphysema, and pulmonary fibrosis. Lung Cancer 2017;111:88-95.

41. Zhang G, Zhang D, Wu J, et al. Low Serum Levels of PreSurgical Total Cholesterol are Associated with Unfavorable Overall Survival in Patients with Operable Non-Small Cell Lung Cancer. Clin Lab 2018;64:321-7.

Cite this article as: Zhang $\mathrm{C}$, Li XK, Cong ZZ, Zheng C, Luo C, Xie K, Xu Y, Gu WF, Qiang Y, Shen Y. Controlling nutritional status is a prognostic factor for patients with lung cancer: a systematic review and meta-analysis. Ann Palliat Med 2021;10(4):3896-3905. doi: 10.21037/apm-20-2328 